Head and Neck Cancer

\title{
Importance of Ki-67 Labeling in Oral Leukoplakia with Features of Dysplasia and Carcinomatous Transformation: An Observational Study over 4 Years
}

\author{
Krishnendu Mondal ${ }^{1}$ Rupali Mandal ${ }^{1}$ Badal Chandra Sarkar ${ }^{2}$ \\ ${ }^{1}$ Department of Pathology, North Bengal Medical College and \\ Hospital, Darjeeling, West Bengal, India \\ ${ }^{2}$ Department of Oral Pathology, North Bengal Dental College and

\begin{abstract}
Address for correspondence Krishnendu Mondal, MD (Pathology), Vill- Fularhat, P.O. \& P.S. Sonarpur, Dist- South 24 Parganas 700150,
\end{abstract} \\ West Bengal, India (e-mail: krishnendu.kriss@gmail.com).
} Hospital, Darjeeling, West Bengal, India

South Asian J Cancer:2020;9:99-104

\begin{abstract}
Keywords

- immunohistochemistry

- Ki-67

- oral epithelial dysplasia

- oral leukoplakia

- squamous cell carcinoma
\end{abstract}

Background Early detection of dysplastic changes within oral potentially malignant disorders is the mainstay to prevent oral cancer. $\mathrm{Ki}-67$ is one of the most useful antigens in this purpose.

Aims The study aims were to recognize and mutually compare the proliferative status of idiopathic oral leukoplakia (OL) patches, which presented through different forms of dysplasia and carcinoma.

Settings and Design In 4 years of observation, cumulatively $140 \mathrm{OL}$ lesions were included for examination. The wholesome Ki-67 labeling scores in each of the subgroups were calculated.

Subjects and Methods The World Health Organization recommended histopathological classification was used to categorize the dysplastic and malignant lesions. Paraffin-embedded tissue sections were processed for Ki-67 immunostaining. The labeling indices (LIs) were quantified semiquantitatively at the site of maximal reactive cells on tissue sections.

Statistical Analysis The statistical comparison was performed by means of the SPSS software (Version 16.0 SPSS Inc.). A p-value $<0.05$ was considered as the benchmark for statistical significance.

Results A steady and significant increment in Ki-67 expression was discovered from dysplastic to malignant OL patches compared with normal mucosa. The labeling differences were significant between normal mucosa and mild dysplasia, as well as between mild, moderate, and severe dysplasia. However, the expression did not differ significantly with the severity of oral cancers.

Conclusions $\mathrm{Ki}-67$ is a useful molecular marker of carcinogenesis in OL. It also serves worthwhile in separating marginally dysplastic lesions, such as mild dysplasia or verrucous carcinoma from their benign epigones.
DOI https://doi.org/ $10.1055 / \mathrm{s}-0040-1721212$ ISSN 2278-330X. (c) 2020. Medlntel Services Pvt Ltd.

This is an open access article published by Thieme under the terms of the Creative Commons Attribution-NonDerivative-NonCommercial-License, permitting copying and reproduction so long as the original work is given appropriate credit. Contents may not be used for commercial purposes, or adapted, remixed, transformed or built upon. (https://creativecommons.org/licenses/by-nc-nd/4.0/) Thieme Medical and Scientific Publishers Pvt. Ltd., A-12, 2nd Floor, Sector 2, Noida-201301 UP, India 


\section{Introduction}

The 13 different centers of population-based cancer registry in India calculated oral cancer as the third most prevalent malignancy after carcinoma breast and carcinoma cervix. Nearly 90 to $95 \%$ of oral cancers consist of squamous cell carcinoma (SCC). Its age-standardized incidence rate has been 12.6 per 100,000 population, which encompasses approximately $30 \%$ Indians suffering from cancer. ${ }^{1,2}$ Approximately $80 \%$ of oral SCC develop from any preexisting potentially malignant disorder (PMD). Oral leukoplakia (OL) is the frequent most PMD encountered in India, with a prevalence of 0.2 to $5.2 \%$, and up to $10 \%$ of malignant transformation rate. ${ }^{1}$

The physiological proliferative activity of oral epithelium is limited to its parabasal layer, the two-cell-thick-strip immediately overlying the basal layer. On dysplastic transformation abnormally, excessive cellular proliferation ensues and the physiological limitations are then breached. Multiple upregulated proliferation markers can thus be useful in determining the dysplastic transition in oral PMDs. ${ }^{3}$ Ki-67 is the best marker to demonstrate the proliferative fraction of oral epithelium. Its nuclear expression is restricted to the G1-S-G2 phases of cell cycle and mitosis. Its short half-life makes it undetectable beyond the proliferative stage. Furthermore, Ki-67 holds the added advantage for the remaining uninfluenced from any extracellular biological altercations. Therefore, Ki-67 not only detects the hyperactive cells in oral epithelial dysplasia (OED), but also its quantitative positivity is comparable to the clinical course or prognostication of the disease.,3 Previously, several researchers evaluated the percentage reactivity of Ki-67 in different histological grades of OED, ${ }^{3-7}$ whereas a handful number of studies incorporated the Ki-67 labeling index (LI) into a simple scoring system for the purpose of comparative ease. ${ }^{8,9} \mathrm{In}$ this latest approach, we focused on the corroboration of Ki-67 labeling scores with multitudes of OED and epithelial malignancy, which clinically presented as idiopathic OL.

\section{Subjects and Methods}

It was a 4-year prospective study orchestrated from January 2013 to December 2016. At the Department of Oral Pathology, North Bengal Dental College and Hospital, idiopathic OL patches are routinely biopsied on an outdoor basis. Subsequent histopathological and immunohistochemical proceedings are performed at the Department of Pathology, North Bengal Medical College and Hospital. Here, during this study, the de novo cases of idiopathic OL that featured dysplastic or carcinomatous changes on histopathology were only analyzed. Persons with a prior history of similar or any other oral PMD, and also in whom therapy has already been commenced for the present patch, were left out. Twenty specimens of normal-appearing buccal mucosa were included from the archives as controls. In accordance with the World Health Organization (WHO) classification, the lesions were diagnosed as mild, moderate, and severe dysplasia, SCC, and verrucous carcinoma (VC). The lesions of SCC in situ were clustered together with severe dysplasia. The SCC lesions were further subclassified into well-differentiated
(WDSCC), moderately differentiated (MDSCC), and poorly differentiated (PDSCC) spectrums. ${ }^{10}$

Ki-67 immunohistochemical (IHC) staining was performed with formalin-fixed paraffin-embedded tissue-sections using 3,3 $\alpha$-diaminobenzidine tetrahydrochloride as a chromogen. Positive and negative controls were devised from the sections of normal tonsil tissue and by omitting the step with primary antibody, respectively. Any recognizable nuclear browning regardless of its intensity was qualified for positivity. Ki-67-positive cells were enumerated at the segments fetching maximal reactive cells. Next, the percentages of overall Ki-67 positive cells in respect of the absolute amount of epithelial cells were determined semiquantitatively. Finally, the Ki67 LI was computed in four scoring categories as follows: $0=0-5 \%, 2=6-25 \%, 4=26-60 \%$, and $6=61-99 \%{ }^{8,9}$ For statistical comparison, chi-square test, as incorporated with SPSS software Version 16.0 (SPSS Inc., Chicago, Illinois, United States) for Windows, was utilized. Statistical significance was inflicted upon $p$-value regressing under 0.05 .

\section{Results}

A total of 786 idiopathic OL patches were biopsied during the observed 4 years. Thereof, 126 patches were histopathologically diagnosed with dysplasia and 14 patches turned out as definite malignancies. Besides SCC (12 cases), 2 cases of VC were recognized ( - Fig. 1). On Ki-67 IHC, all 20 normal mucosal samples were labeled as score 0 , as was also evidenced with 17 out of 64 patches of mild dysplasia. The remaining mildly dysplastic lesions expressed LI scores of 2 (26 cases) and 4 (21 cases) ( - Fig. 2). Henceforth, a significant labeling discrepancy $(p<0.0001)$ was noted between normal and mildly dysplastic oral mucosae ( - Fig. 1 ).

The lesions featuring moderate and severe dysplasia were immunohistochemically distributed into scores 2,4 , and 6 (-Figs. 3 and 4). Their labeling diversity fetched a low statistical significance $(p=0.012)$ in this regard. However, the overall intensifying Ki-67 LI scores in respect of worsening histological grades of dysplasia garnered significant statistical association with $p<0.0001$ ( - Fig. 1).

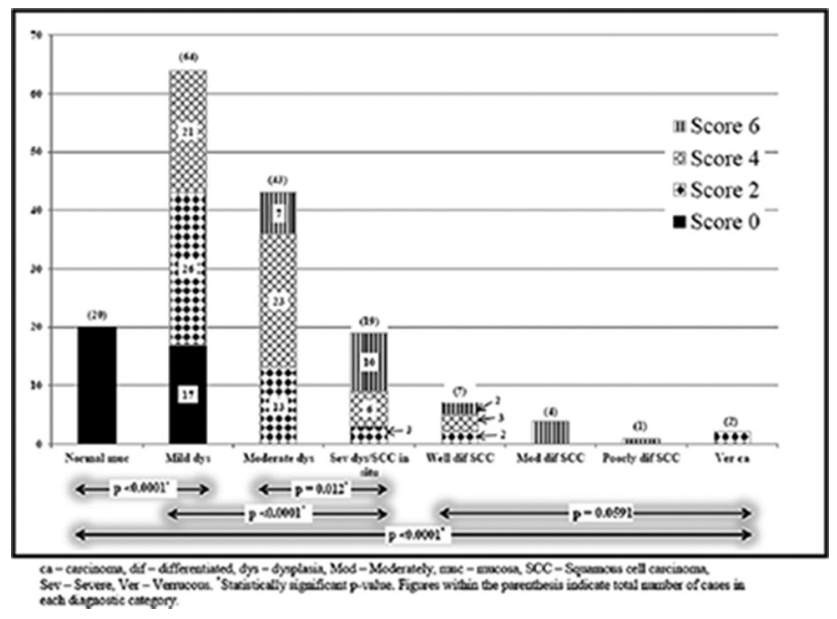

Fig. 1 Comparison of Ki-67 labeling in normal oral mucosa, oral epithelial dysplasia, and carcinoma. 

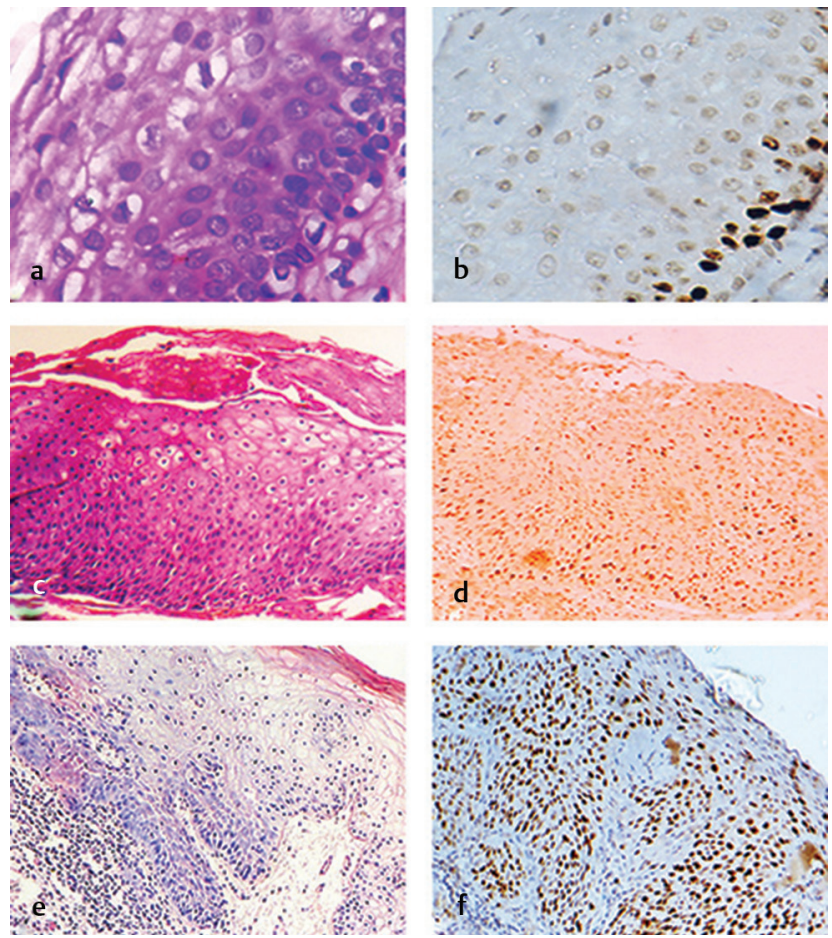

Fig. 2 Representative histopathological and immunohistochemical (IHC) images of mild dysplasia: (a, b) score 0 ; $(\mathbf{c}, \mathbf{d})$ score 2 ; $(\mathbf{e}, \mathbf{f})$ score 4. (a) Hematoxylin and eosin (H\&E), x400. (c, e) H\&E, x100. (b) Ki-67 IHC, x400. (d, f) Ki-67 IHC, x100.
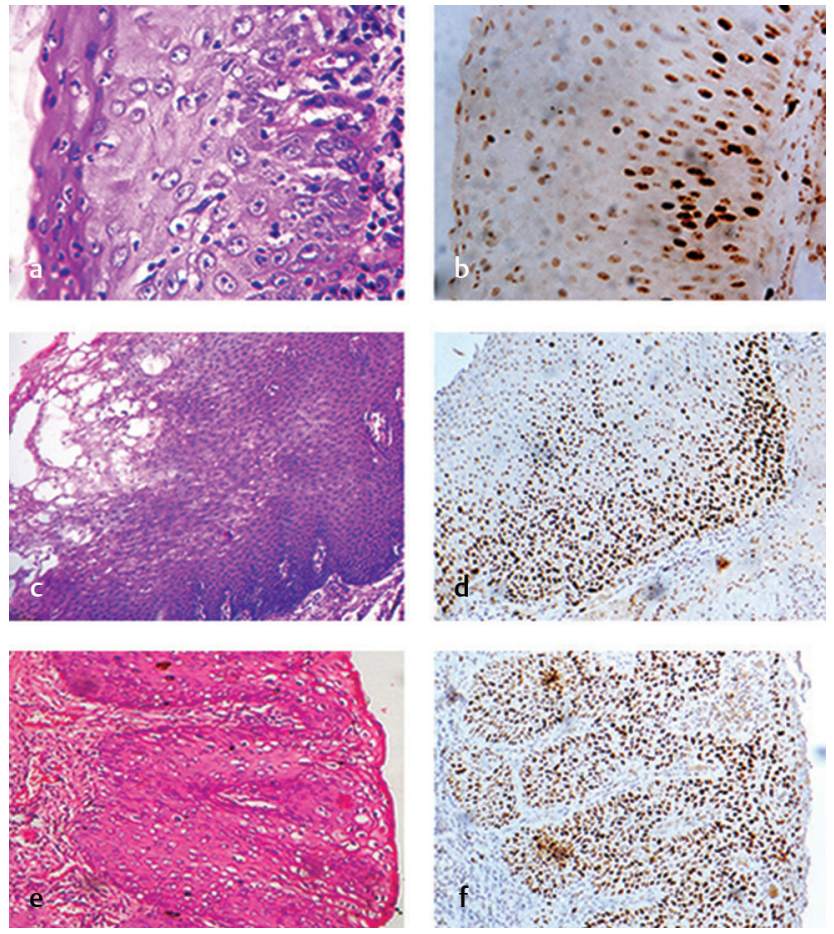

Fig. 3 Representative histopathological and immunohistochemical (IHC) images of moderate dysplasia: (a, b) score 2; (c, d) score 4; (e, f) score 6. (a) Hematoxylin and eosin (H\&E), x400. (c, e) H\&E, x100. (b) Ki-67 IHC, x400. (d, f) Ki-67 IHC, x100.
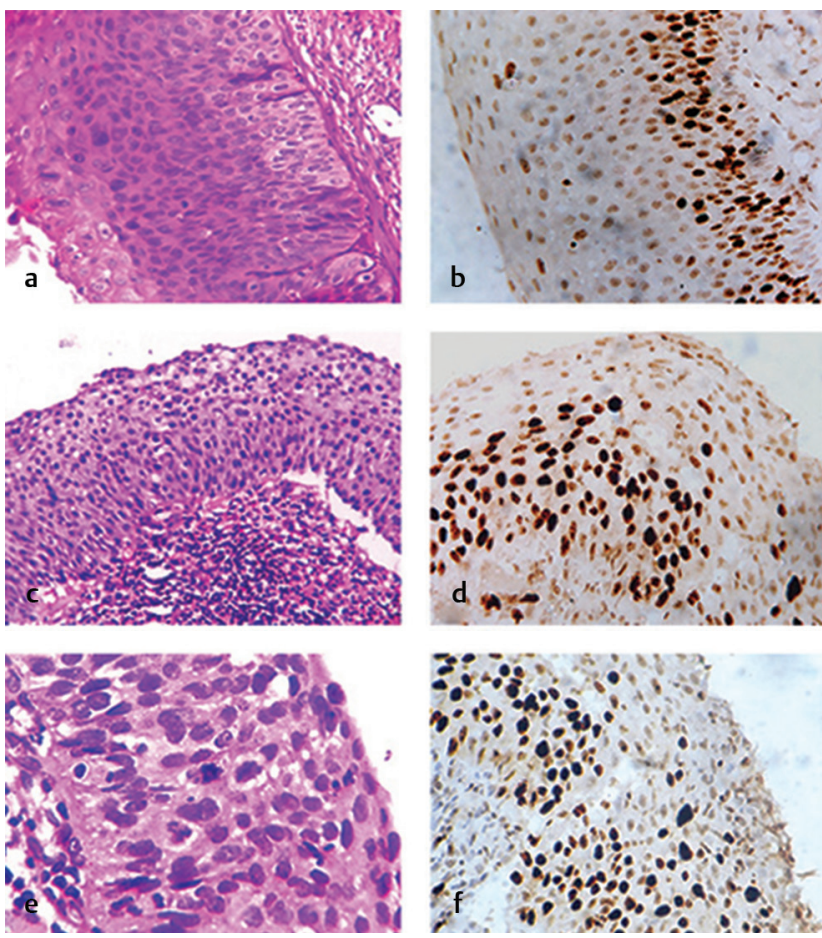

Fig. 4 Representative histopathological and immunohistochemical (IHC) images of severe dysplasia/squamous cell carcinoma in situ: (a, b) score 2; (c, d) score 4; (e, f) score 6 (a, c) Hematoxylin and eosin (H\&E), x100. (e) H\&E, x400. (b, d, f) Ki-67 IHC, x100.
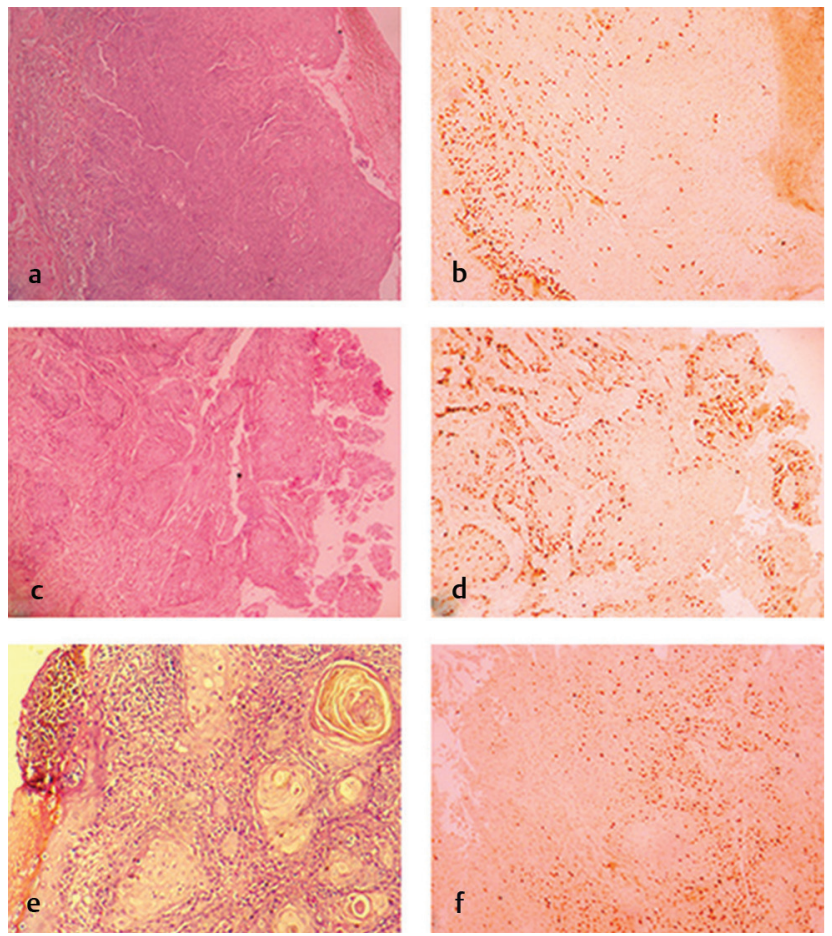

Fig. 5 Representative histopathological and immunohistochemical (IHC) images of well-differentiated squamous cell carcinoma: (a, b) score 2; (c, d) score 4; (e, f) score 6. (a, c, e) Hematoxylin and eosin (H\&E), x100. (b, d, f) Ki-67 IHC, x100. 

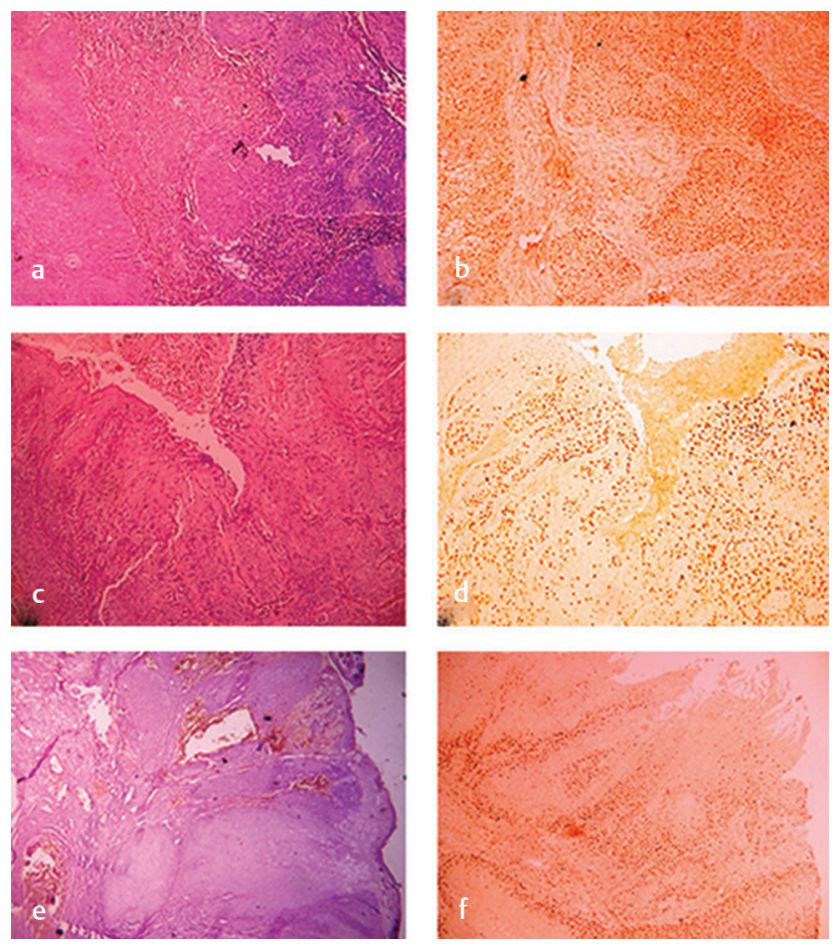

Fig. 6 Representative histopathological and immunohistochemical (IHC) images of $(\mathbf{a}, \mathbf{b})$ moderately differentiated squamous cell carcinoma, (c, d) poorly differentiated squamous cell carcinoma, and (e, f) verrucous carcinoma. (a, c) Hematoxylin and eosin (H\&E), x100. (e) H\&E, x40. (b, d) Ki-67 IHC, x100. (f) Ki-67 IHC, x40.

The seven cases of WDSCCs expressed LI scores of 2 to 6 in near-equivalent distributions (-Fig. 5). Alongside, all cases of MDSCCs and PDSCCs were labeled as score 6. Both lesions of VC conveyed a mere score 2 on Ki-67 labeling (-Fig. 6). This difference among carcinomatous lesions did not generate any statistical significance $(p=0.0591)$. Although on ultimate comparison, a statistically significant association of escalating Ki-67 LI scores was found between normal oral mucosae, through various grades of OED, up to multitudes of oral epithelial malignancies $(p<0.0001)$ (-Fig. 1).

\section{Discussion}

The normal biological proliferation within stratified squamous epithelium is maintained by the stem cells at the basal layer and their direct progenies at the parabasal layer. Its regulatory proteins are delivered through the underlying connective tissue, whereas its synchronized differentiation toward surface is nourished by the factors produced from keratinocytes. In PMDs, abnormal cell proliferation commonly prevails. The synchronicity in mucosal maturation is lost. Even the suprabasal squamous cells are then capable of proliferating independently from their regulatory molecules. ${ }^{4}$ The causative genetic derangements lead to the expression of certain molecular markers that apparently correspond with different grades of OED. These markers are classified into: (1) genomic markers, for example, p53;
(2) proliferation markers, for example, Ki-67; and (3) differentiation markers, for example, CK13 and CK17. ${ }^{11}$

p53 and Ki-67 are the most widely implemented molecular antigens to discriminate OED from normal oral mucosa. The p53 is a tumor suppressor gene, and its resultant protein is normally expressed at the G1 phase. On its mutation, the protein resumes a longer half-life. Therefore, abnormal suprabasal expression of p53 is an important indicator of oral carcinogenesis.6,8,11 However, sometimes, a defective catabolism or binding with other proteins can also prompt its intranuclear precipitation. Several researchers derived low positive predictive value of independent p53 expression in this context. On the contrary, Ki-67 is bereft of such limitations, which makes it an individualistic proliferation marker. It tallies with the existence as well as the virulence of dysplasia. Often, it denotes the aggressiveness of an already invasive SCC and also the proliferative activity of neoplastic cells at the edge of infiltrating fronds. Such a phenomenal upregulation of Ki-67 in dysplastic and carcinomatous epithelium makes it an unparalleled prognostic marker., ${ }^{9,12,13}$ Otherwise, only CK13, with its loss of expression beyond severe dysplasia, is comparable to Ki-67 for independent prognostic significance. ${ }^{14}$ Other anticipated antigens such as pl6INK4a, CK17, bcl-2, proliferating cell nuclear antigen, cyclooxygenase-2, and cyclin D1 have mostly been considered as an adjunct to Ki-67 IHC., 71-14

In all the 20 normal mucosal samples from this study, the Ki-67-positive cells were sprinkled within its basal and parabasal layers only. With the progression of dysplasia till SCC, these cells gradually grew more populous at the suprabasal epithelium. Such trend of Ki-67 reactivity in OED or oral SCC has been discussed previously by many researchers., ${ }^{72-14}$ But unlike theirs, in this study, the OL patches that did not exhibit any dysplasia were excluded. Instead, we solely concentrated upon the dysplastic and malignant patches. Therein, a statistically significant difference of proliferative activity was procured between normal mucosa, OED, and SCC (- Fig. 1).

Earlier, many researchers did fail to find any significant difference in Ki-67 labeling between normal and mildly dysplastic oral lesions. Often, these both were incorporated together within the same low-risk category for statistical comparison. It might have been potential pitfalls of their limited sample size. ${ }^{3,4}$ In the discussed study, the Ki-67 labeling pattern of mild dysplasia significantly differed from normal epithelium. Compared with all the normal tissues being scored as 0 , 26 out of the 64 mild dysplasia lesions were labeled as score 2 , and 21 lesions were labeled as score 4 (-Fig. 1). In this situation, the 17 samples with score 0 could possibly be representatives of reactive atypical epithelium..$^{14}$ Raju et al ${ }^{12}$ in 2005 and, more recently, Sinanoglu et $\mathrm{al}^{7}$ also computed a similar kind of statistical comparison as of ours.

Multiple antecedent studies suggested that the abnormal Ki-67 LI corresponds best with moderate dysplasia and the lesions beyond. A significant labeling difference between low-grade and high-grade dysplasia is almost always there. Though sometimes, the researchers could not segregate 
moderate and severe dysplasia on the basis of Ki-67 labeling. They preferentially clubbed these two lesions for comparative ease. ${ }^{3,414,15}$ Exceptional reports suggesting an indifferent proliferative activity at different stages of OED are also documented., ${ }^{5,12} \mathrm{Ki}-67$ labeling according to the WHO-defined trimodal grades of $\mathrm{OED}^{10}$ has only been reminisced by a handful number of studies ahead of our present deliberation. Similarly, Kumar et al examined 19, 7, and 3 samples of mild, moderate, and severe dysplasia, respectively. There was a significantly high proliferative activity in severe > moderate > mild dysplasia. ${ }^{6}$ Sinanoglu et al recognized the three phases of dysplasia as oral intraepithelial neoplasia (OIN) subgroups 1 to 3 . The Ki-67 expression within their OIN1 and OIN2 cases was nearly the same. Neither did the overall exaggerating $\mathrm{LI}$ from OIN1 $\rightarrow \mathrm{OIN} 2 \rightarrow \mathrm{OIN} 3$ gather any statistical significance. Still, there was a significantly high Ki-67 expression in OIN3 compared with OIN2. ${ }^{7}$ Identically, the Ki-67 LI in this study steadily aggravated in a statistically significant way from mild through moderate up to severe dysplasia. The isolated variation of LI between moderate and severe dysplasia was also significant ( - Fig. 1). Because of such heterogeneous observations in this particular context, additional research attributes are still required to reach a definite opinion.

Normally, the Ki-67 responsiveness enhances through the progressive grades of oral SCC. In WDSCC, the proliferative activity is localized at the periphery of the tumoral fronds. Centrally, the tumor nests mature into frequent squamous pearls and lack Ki-67 expression. Onto MDSCC, the Ki-67-reactive cells sporadically begin to populate more centrally as much at the edge of tumor nests. The relatively undifferentiated neoplastic cells in PDSCC express more diffuse staining pattern. ${ }^{3,16-18}$ The presently described report was no different either. Herein, the seven WDSCC cases acquired LI scores of 2 (two cases), 4 (three cases), and 6 (two cases). However, all the lesions of MDSCC and PDSCC were labeled as score 6. Despite this apparently improving Ki-67 expression in respect of diminutive differentiation of oral SCC, the same did not manage any statistical significance. Similar kind of insignificant proliferative activity among oral cancers has already been reiterated by Raju et al. ${ }^{12}$ Under similar situation, Birajdar et $\mathrm{al}^{4}$ and Piffkó et $\mathrm{al}^{19}$ achieved a strange response. The Ki-67 labeling in their examined MDSCC patches fell short of WDSCC, which then again augmented to a significant high in PDSCC cases. In fact, the histological grades of oral SCC bear a dismal correlation with its prognosis, and this nature of unworthy proliferative discrepancies within oral cancers could just be the reason behind. ${ }^{10}$

VC is a locally invasive low-grade subtype of SCC. It is characterized by an expansile bulbous downgrowth of mature squamous epithelium penetrating into the submucosa. The irregular stromal infiltration seen in classic SCC is absent. Metastasis also never happens with VC. ${ }^{20}$ Its closest mimickers are verrucous hyperplasia $(\mathrm{VH})$ and WDSCC. Ki-67 IHC can unanimously discriminate VC from VH. The reactive cells in VC are localized at the basal and the suprabasal regions in the lower half of the epithelium, whereas in $\mathrm{VH}$, these positive cells are strictly confined within the basal layer just as good as the normal mucosa. ${ }^{20,21}$ Rather, conflicting opinions are there regarding the role of $\mathrm{K}_{\mathrm{i}}-67$ IHC in the separation of VC from WDSCC. Previously, Saito et $\mathrm{al}^{22}$ and Adegboyega et $\mathrm{al}^{23}$ considered the presence of positive cells throughout the entire epithelial thickness as the principal discriminating point in favor of WDSCC. Contradictorily, Zargaran et $\mathrm{al}^{21}$ did not find this way-out any helpful. Whatsoever, such dilemma did not surface in this study. None of the examined two VC patches did comingle with VH or WDSCC either histologically or immunohistochemically. In WDSCC, the Ki-67-positive cells were scattered along full thickness of the epithelium, and in VC, these cells were restricted within the suprabasal 4 to 6 layers only (-Figs. 5 and $\mathbf{6}$ ).

Conclusively, this latest study implements that a significantly escalating Ki-67 immunoexpression along with increasing grades of dysplasia and furthermore into the carcinoma actually makes it a predictive marker for cancerous transformation in OL. Its differential overexpression in various grades of dysplasia beholds the necessity of WHO-recommended tripartite classification for dysplasia. Ki-67 IHC is of particular importance in mild dysplasia and VC, where it clearly secludes the erroneously included reactive atypical epithelium. Reciprocally, an insignificant labeling difference within multimodal oral carcinomas argues the negligible correlation between its histological grades and outcome.

\section{Conclusion}

Therewith Ki-67 is conclusively depicted as an important immunohistochemical marker for dysplastic and malignant transformation in OL, and also its credibility toward discrimination of mildly dysplastic patches and VCs from reactive epithelial atypia has been ratified.

\section{Funding}

None.

\section{Conflicts of Interest}

There are no conflicts of interest.

\section{References}

1 Sridharan G. Epidemiology, control and prevention of tobacco induced oral mucosal lesions in India. Indian J Cancer 2014;51(1):80-85

2 Takiar R, Krishnan SK, Shah VP. A model approach to calculate cancer prevalence from 5 years survival data for selected cancer sites in India-part II. Asian Pac J Cancer Prev 2014;15(14):5681-5684

3 Dwivedi N, Chandra S, Kashyap B, Raj V, Agarwal A. Suprabasal expression of Ki-67 as a marker for the severity of oral epithelial dysplasia and oral squamous cell carcinoma. Contemp Clin Dent 2013;4(1):7-12

4 Birajdar SS, Radhika M, Paremala K, Sudhakara M, Soumya M, Gadivan M. Expression of Ki-67 in normal oral epithelium, leukoplakic oral epithelium and oral squamous cell carcinoma. J Oral Maxillofac Pathol 2014;18(2):169-176

5 Reddy VM, Saxena S. Assessment of Ki-67 in healthy, premalignant, and malignant lesions of oral mucosa and its predictive role in disease progression. Int J Oral Med Sci 2010;9:67-74 
6 Kumar P, Kane S, Rathod GP. Coexpression of p53 and Ki 67 and lack of c-erbB2 expression in oral leukoplakias in India. Braz Oral Res 2012;26(3):228-234

7 Sinanoglu A, Soluk-Tekkesin M, Olgac V. Cyclooxygenase-2 and Ki67 expression in oral leukoplakia: a clinicopathological study. J Oral Maxillofac Res 2015;6(2):e3

8 Kannan S, Chandran GJ, Pillai KR, et al. Expression of p53 in leukoplakia and squamous cell carcinoma of the oral mucosa: correlation with expression of Ki67. Clin Mol Pathol 1996;49(3):M170-M175

9 Humayun S, Prasad VR. Expression of p53 protein and ki-67 antigen in oral premalignant lesions and oral squamous cell carcinomas: an immunohistochemical study. Natl J Maxillofac Surg 2011;2(1):38-46

10 Barnes L, Eveson JW, Reichart P, Sidransky D, eds. WHO Classification of Tumours. Pathology \& Genetics of Head and Neck Tumours. Lyon: IARC Press; 2005

11 Salati NA. Diagnostic potential of epithelial biomarkers in oral diseases-lmmunohistochemical basis. IOSR J Dent Med Sci 2013;5:37-40

12 Raju B, Mehrotra R, Oijordsbakken G, Al-Sharabi AK, Vasstrand EN, Ibrahim SO. Expression of p53, cyclin D1 and Ki-67 in pre-malignant and malignant oral lesions: association with clinicopathological parameters. Anticancer Res 2005;25(6C):4699-4706

13 Nasser W, Flechtenmacher C, Holzinger D, Hofele C, Bosch FX. Aberrant expression of p53, p16INK4a and Ki-67 as basic biomarker for malignant progression of oral leukoplakias. J Oral Pathol Med 2011;40(8):629-635

14 Yagyuu T, Obayashi C, Ueyama Y, et al. Multivariate analyses of Ki-67, cytokeratin 13 and cytokeratin 17 in diagnosis and prognosis of oral precancerous lesions. J Oral Pathol Med 2015;44(7):523-531

15 Warnakulasuriya S, Reibel J, Bouquot J, Dabelsteen E. Oral epithelial dysplasia classification systems: predictive value, utility, weaknesses and scope for improvement. J Oral Pathol Med 2008;37(3):127-133

16 Kumar KV, Chaithanya KH, Punde P, Thorat A, Jangam AG, Deepthi S. Comparative evaluation of imunohistochemical expression of Ki-67 in oral lichen planus, oral leukoplakia and normal mucosa cases. J Int Oral Health 2015;7:82-87

17 Dissanayake U, Johnson NW, Warnakulasuriya KA. Comparison of cell proliferation in the centre and advancing fronts of oral squamous cell carcinomas using Ki-67 index. Cell Prolif 2003;36(5):255-264

18 Vieira FL, Vieira BJ, Guimaraes MA, Aarestrup FM.. Cellular profile of the peritumoral inflammatory infiltrate in squamous cells carcinoma of oral mucosa: correlation with the expression of Ki67 and histologic grading. BMC Oral Health 2008;8-25

19 Piffkó J, Bánkfalvi A, Ofner D, et al. In situ assessment of cell proliferation at the invasive front of oral squamous cell carcinomas. Virchows Arch 1996;429(4-5):229-234

20 Klieb HB, Raphael SJ. Comparative study of the expression of p53, Ki67, E-cadherin and MMP-1 in verrucous hyperplasia and verrucous carcinoma of the oral cavity. Head Neck Pathol 2007;1(2):118-122

21 Zargaran M, Eshghyar N, Baghaei F, Moghimbeigi A. Assessment of cellular proliferation in oral verrucous carcinoma and well-differentiated oral squamous cell carcinoma using Ki67: a non-reliable factor for differential diagnosis? Asian Pac J Cancer Prev 2012;13(11):5811-5815

22 Saito T, Nakajima T, Mogi K. Immunohistochemical analysis of cell cycle-associated proteins p16, pRb, p53, p27 and Ki-67 in oral cancer and precancer with special reference to verrucous carcinomas. J Oral Pathol Med 1999;28(5):226-232

23 Adegboyega PA, Boromound N, Freeman DH. Diagnostic utility of cell cycle and apoptosis regulatory proteins in verrucous squamous carcinoma. Appl Immunohistochem Mol Morphol 2005;13(2):171-177 